\title{
Efeito da brimonidina na circulação retrobulbar em pacientes com glaucoma - estudo com Doppler colorido
}

\author{
Effect of brimonidine on the retrobulbar circulation in glaucomatous patients - \\ a color Doppler study
}

\author{
Maria Rosa Bet de Moraes Silva ${ }^{1}$ \\ Hamilton Almeida Rollo² \\ Silvana A. Schellini ${ }^{3}$
}

Trabalho realizado na Faculdade de Medicina de Botucatu - Universidade Estadual Paulista (UNESP). ${ }^{1}$ Professor Assistente Doutor do Departamento de Oftalmologia, Otorrinolaringologia e Cirurgia de Cabeça e Pescoço - Universidade Estadual Paulista (UNESP) - Botucatu.

${ }^{2}$ Professor Assistente Doutor do Departamento de Cirurgia e Ortopedia - Universidade Estadual Paulista (UNESP) - Botucatu.

${ }^{3}$ Professor Livre-docente do Departamento de Oftalmologia, Otorrinolaringologia e Cirurgia de Cabeça e Pescoço - Universidade Estadual Paulista (UNESP) Botucatu.

Endereço para correspondência: Maria Rosa Bet de Moraes Silva - Dep. OFT/ORL/CCP - Faculdade de Medicina de Botucatu - Universidade Estadual Paulista (UNESP) - Botucatu (SP) Brasil - CEP 18618-000. E-mail rosabet@fmb.unesp.br

\begin{tabular}{|l|}
\hline RESUMO \\
\hline Objetivo: Avaliar o efeito do tartarato de brimonidina a $0,2 \%$ tópico, ins- \\
tilado de $12 / 12$ horas, na circulação retrobulbar em pacientes portadores \\
de glaucoma. Métodos: Foram estudados os 2 olhos de 16 pacientes \\
portadores de glaucoma primário de ângulo aberto e glaucoma crônico de \\
ângulo estreito com iridotomia. Usando o Doppler colorido foram avali- \\
ados: velocidade sistólica máxima, velocidade diastólica final e índice de \\
resistência das artéria central da retina, artéria ciliar posterior curta \\
temporal e artéria oftálmica. As avaliações foram feitas antes e depois do \\
uso da brimonidina. Resultados: O tartarato de brimonidina a $0,2 \%$ \\
aumentou significativamente a velocidade sistólica máxima $(28,24$ para \\
34,23 cm/seg) e velocidade diastólica final $(6,62$ para 8,10 cm/seg) no olho \\
direito e reduziu o índice de resistência ( 0,75 para 0,71$)$ no olho esquerdo \\
da artéria oftálmica. Conclusão: O tartarato de brimonidina $0,2 \% 2 x /$ dia \\
aumentou significativamente a velocidade sistólica máxima e velocidade \\
diastólica final e reduziu índice de resistência da artéria oftálmica de \\
pacientes glaucomatosos. Este efeito sugere que a brimonidina pode \\
beneficiar pacientes glaucomatosos com insuficiência vascular na cabe- \\
ça do nervo óptico.
\end{tabular}

Descritores: Disco óptico/irrigação sangüínea; Glaucoma; Quinoxalinas/uso terapêutico; Ultra-sonografia Doppler em cores

\section{INTRODUÇ̃̃̃O}

A pressão intraocular (PIO) aumentada é importante fator de risco para glaucoma, mas a patogênese do dano glaucomatoso na cabeça do nervo óptico (NO) não está ainda bem compreendida, havendo evidências de que fatores vasculares estão envolvidos nesta patogênese, pelo menos em alguns pacientes ${ }^{(1-2)}$.

O tartarato de Brimonidina a $0,2 \%$ é droga alfa- 2 agonista altamente seletiva $^{(3)}$ eficaz em reduzir a PIO em pacientes com glaucoma ${ }^{(4)}$. Mas é importante que drogas utilizadas no tratamento do glaucoma, além de reduzirem a PIO não apresentem efeitos adversos no fluxo sangüíneo ocular ${ }^{(5)}$.

Embora a vasoconstricção seja um efeito alfa-1, a seletividade de nenhuma droga é totalmente específica ${ }^{(3)}$ podendo depender da concentração, das características do leito vascular, do tamanho do vaso e da espécie animal estudada. Assim é importante excluir a possibilidade de qualquer efeito vascular adverso de drogas utilizadas para o tratamento do glaucoma, particularmente ao nível da cabeça do NO.

Este estudo foi realizado para avaliar os efeitos da administração do 
tartarato de brimonidina a $0,2 \%$ tópico, duas vezes ao dia, na circulação retrobulbar de pacientes com glaucoma.

\section{MÉTODOS}

Foram avaliados prospectivamente 16 pacientes com glaucoma crônico (glaucoma primário de ângulo aberto e glaucoma crônico de ângulo fechado com iridotomia) da clínica de glaucoma do Hospital das Clínicas da Faculdade de Medicina de Botucatu - UNESP. O tartarato de Brimonidina a 0,2\% (Alphagan $^{\circledR}$, Allergan-Frumtost) foi instilado $2 \mathrm{x} /$ dia por pelo menos 15 dias, em associação ou como tratamento único. Foram estudados ambos os olhos dos pacientes.

Foram critérios de exclusão: olhos com história de uveítes, olhos submetidos à cirurgia intra-ocular prévia incluindo cirurgia para glaucoma e olhos que necessitaram de cirurgia anti-glaucomatosa durante o estudo.

Foi obtido em todos os casos, termo de consentimento livre e esclarecido e o trabalho foi submetido e aprovado pela Comissão de Ética Médica da Faculdade de Medicina de Botucatu - UNESP.

As avaliações da circulação retrobulbar com Doppler colorido foram sempre realizadas no período da tarde entre 14 e 16 horas. Foram realizadas 2 avaliações para cada paciente, antes do tratamento e após o mesmo, que variou de 15 dias a 4 meses.

Todas as avaliações com Doppler colorido foram realizadas por um sonografista experiente (HAR) que desconhecia a condição clínica do paciente quanto à medicação antiglaucomatosa utilizada. A avaliação foi realizada com o aparelho Philips Platinum (Philips Medical Systems - Santa Ana - Califórnia) com sonda linear de 7,5 MHz. Todas as avaliações foram feitas com paciente deitado em posição supina.

Foram avaliadas: velocidade sistólica máxima (VSM) e velocidade diastólica final (VDF) na artéria oftálmica, na artéria central da retina e na artéria ciliar curta posterior temporal. O índice de resistência (IR) foi calculado para cada vaso de acordo com a fórmula:

$$
\frac{\text { VSM-VDF }}{\text { VSM }}
$$

O ângulo de insonação, isto é, o ângulo formado pelo eixo das ondas ultra-sonográficas e a direção do sangue, foi corrigido para cada vaso e o volume de amostragem (área gerada eletronicamente pelo aparelho para analisar a curva espectral) escolhido, foi de $0,6 \mathrm{~mm}$.

Foram também avaliados a pressão intra-ocular (PIO) com tonômetro de aplanação de Goldmann e a pressão arterial sistólica e diastólica (PAS e PAD) antes de cada avaliação com Doppler colorido.

Foi realizada análise estatística por meio do teste $\mathrm{t}$ de Student para amostras pareadas para os diversos parâmetros estudados: PIO, PAS, PAD e VSM, VDF e IR nos 3 vasos estudados e separadamente para olhos esquerdos e direitos. Foram considerados significativos valores de $\mathrm{P}<0,05 \%$.

\section{RESULTADOS}

Foram estudados 6 homens e 10 mulheres. A média da idade dos pacientes foi de 56,82 $\pm 13,7$ anos ( 30 a 77 anos). Quanto à raça 12 eram brancos, 3 negros e 1 amarelo.

Dez pacientes usavam drogas hipotensoras sistêmicas (sendo que 1 usava propranolol), 3 hipoglicemiantes orais e 2, hormônios para menopausa.

Dez pacientes estavam em uso de timolol, 3 timolol e pilocarpina e 1 paciente timolol e latanoprost, quando foi introduzida a Brimonidina. Os outros 2 pacientes foram tratados apenas com Brimonidina.

$\mathrm{O}$ tratamento com as outras drogas que não a Brimonidina foi mantido inalterado depois da introdução da mesma.

A PIO média \pm DP foi de 17,20 $\pm 3,68 \mathrm{mmHg}$ antes e de $15,50 \pm$ $1,18 \mathrm{mmHg}$ depois do tratamento com brimonidina tópica no olho direito (OD) e de $17,00 \pm 2,86 \mathrm{mmHg}$ antes e $15,42 \pm 2,74 \mathrm{mmHg}$ depois, no olho esquerdo (OE). A PIO foi reduzida de $9,9 \%$ no OD e $9,3 \%$ no OE, mas a redução foi estatisticamente insignificante (Tabela 1).

A PAS média \pm DP foi de $142 \pm 24,9 \mathrm{mmHg}$ antes e de $149 \pm$ $33,7 \mathrm{mmHg}$ depois $(\mathrm{p}=0,24)$. A PAD média \pm DP foi de $89,5 \pm$ $18,8 \mathrm{mmHg}$ antes e de $91,0 \pm 15,8 \mathrm{mmHg}$ depois do tratamento com a Brimonidina $(p=0,75)$. Estas alterações não foram significantes.

A tabela 2 mostra a média dos valores da VSM, VDF e do IR na artéria oftálmica, artéria central da retina e artéria ciliar curta posterior temporal, antes e depois da Brimonidina nos OD e OE.

$\mathrm{Na} A O$ houve aumento significante da VSM e VDF no OD: 28,24 para $34,23 \mathrm{mmHg}(\mathrm{p}<0,01)$ e 6,72 para $8,10 \mathrm{mmHg}(\mathrm{p}<0,01)$ respectivamente e redução no IR no OE de 0,75 para $0,71(p<0,01)$.

Não foram observadas alterações significantes nas VSM, VDF e IR dos demais vasos (Tabela 2).

\section{DISCUSSÃO}

O Doppler colorido tem sido utilizado para avaliação da circulação ocular humana ${ }^{(6)}$. Este método mede a velocidade da circulação dos vasos retrobulbares que fornecem suprimento sangüíneo à cabeça do $\mathrm{NO}$, vasos que são difíceis de observar e que têm grande variação anatômica, especialmente as artérias ciliares posteriores curtas ${ }^{(7)}$. Existem controvérsias quanto à correlação das alterações da velocidade de fluxo detectadas pelo Doppler colorido e o fluxo de sangue no vaso $^{(8)}$. Contudo, há evidências de boa correlação entre a

\begin{tabular}{|c|c|c|c|}
\hline & ANTES & DEPOIS & \\
\hline OD & $17,20 \pm 3,68$ & $15,50 \pm 1,18$ & $p=0,15$ \\
\hline OE & $17,00 \pm 2,86$ & $15,42 \pm 2,74$ & $p=0,19$ \\
\hline \multicolumn{4}{|c|}{ Poder do teste: $\mathrm{OD}=0,18 ; \mathrm{OE}=0,14$} \\
\hline
\end{tabular}




\begin{tabular}{|c|c|c|c|c|c|c|}
\hline & \multicolumn{3}{|c|}{ OD } & \multicolumn{3}{|c|}{$\mathrm{OE}$} \\
\hline & ANTES & DEPOIS & V. de $P$ & ANTES & DEPOIS & V. de $P$ \\
\hline & \multicolumn{6}{|c|}{ ARTÉRIA OFTÁLMICA } \\
\hline VSM & $28,24 \pm 9,32$ & $34,23 \pm 11,21$ & $(0,01)^{*}$ & $27,75 \pm 11,24$ & $28,43 \pm 12,45$ & $(0,76)$ \\
\hline VDF & $6,72 \pm 2,10$ & $8,10 \pm 2,58^{\circ}$ & $(0,01)^{*}$ & $6,84 \pm 2,90$ & $7,93 \pm 3,42$ & $(0,13)$ \\
\hline \multirow[t]{2}{*}{ IR } & $0,75 \pm 0,06$ & $0,76 \pm 0,06$ & $(0,78)$ & $0,75 \pm 0,05$ & $0,71 \pm 0,07$ & $(0,02)^{*}$ \\
\hline & \multicolumn{6}{|c|}{ ARTÉRIA CENTRAL DA RETINA } \\
\hline VSM & $12,77 \pm 2,42$ & $12,85 \pm 2,37$ & $(0,93)$ & $12,32 \pm 2,07$ & $11,90 \pm 2,67$ & $(0,56)$ \\
\hline VDF & $3,43 \pm 0,81$ & $3,70 \pm 1,13$ & $(0,50)$ & $3,53 \pm 0,82$ & $3,38 \pm 0,83$ & $(0,58)$ \\
\hline \multirow[t]{2}{*}{ IR } & $0,73 \pm 0,05$ & $0,71 \pm 0,07$ & $(0,40)$ & $0,70 \pm 0,08$ & $0,71 \pm 0,06$ & $(0,76)$ \\
\hline & \multicolumn{6}{|c|}{ ARTÉRIA CILIAR CURTA POSTERIOR TEMPORAL } \\
\hline VSM & $10,65 \pm 1,98$ & $10,16 \pm 2,31$ & $(0,52)$ & $10,73 \pm 1,73$ & $10,84 \pm 2,55$ & $(0,84)$ \\
\hline VDF & $3,62 \pm 0,57$ & $3,27 \pm 0,76$ & $(0,21)$ & $3,69 \pm 0,92$ & $3,66 \pm 1,01$ & $(0,94)$ \\
\hline IR & $0,65 \pm 0,07$ & $0,67 \pm 0,06$ & $(0,39)$ & $0,66 \pm 0,09$ & $0,66 \pm 0,07$ & $(0,98)$ \\
\hline
\end{tabular}

velocidade de fluxo e o fluxo sangüíneo nas artérias cerebrais $^{(9)}$.

Como fatores vasculares podem estar envolvidos na patogênese do glaucoma, é importante estudar o efeito de drogas utilizadas para reduzir a PIO, na circulação da cabeça do NO.

Neste estudo com Doppler colorido o tartarato de Brimonidina a $0,2 \%$ aumentou significativamente a VSM e VDF na $\mathrm{AO}$ do $\mathrm{OD}$ e reduziu significativamente o IR na $\mathrm{AO}$ do OE. Não foram observadas alterações significantes nos demais vasos.

Lachkar et al., $1998^{(10)}$, não observaram alterações na circulação retrobulbar com Doppler colorido após uso de tartarato de Brimonidina a $0,2 \%$ em pacientes com hipertensão ocular. Explicam estes resultados pela alta especificidade da Brimonidina pelos receptores adrenérgicos alfa-2. Papaconstantinou et al., $2000^{(11)}$, também não observaram alteração do fluxo coroidal com uso de Brimonidina em pacientes com glaucoma de ângulo aberto. No entanto estes autores utilizaram outro método em seu estudo, o Langham Ocular Blood Flow. Carlsson et al., 2000 $0^{(12)}$, não observaram alterações no fluxo retiniano em pacientes hipertensos oculares após uso da Brimonidina $0,2 \%$. Aqui também os autores utilizaram metodologia diferente, a Scanning Laser Doppler Flowmetry, além da diferença no tipo de paciente, hipertensos oculares.

A Brimonidina é 32 vezes mais seletiva para o receptor alfa2 do que a apraclonidina e 7 vezes mais seletiva do que a clonidina ${ }^{(3)}$. Porém, a brimonidina mesmo muito seletiva, não o é por absoluto, podendo estimular receptores alfa-1 e provocar efeitos mediados pelos mesmos como a vasoconstrição. Spada et al., 1995(13), estudando enxertos retinianos humanos antes e depois da aplicação tópica de Brimonidina, não observaram alterações no calibre dos vasos quando a concentração era de $10 \mathrm{Mm}$ e observaram $8 \%$ de redução do calibre quando a concentração era de $100 \mathrm{Mm}$.

A Brimonidina tópica pode atingir os vasos retrobulbares por absorção sistêmica. Isto é sugerido pelo fato de que olhos contralaterais de olhos tratados com Brimonidina tópica, apresentam queda da $\mathrm{PIO}^{(14)}$.
Pelo exposto acima algum efeito de vasoconstrição poderia ser observado na circulação retrobulbar.

Os resultados deste estudo mostram portanto aumento inesperado na VSM e VDF na AO assim como uma redução no IR no mesmo vaso.

A Brimonidina poderia ter produzido vasodilatação via receptores adrenérgicos alfa- 2 das células endoteliais, que poderiam liberar fatores relaxantes ${ }^{(15-16)}$. No entanto a existência de receptores alfa-2 nos vasos orbitários é duvidosa ${ }^{(13,17-18)}$.

Outra possibilidade para explicar estes resultados seria a influência de drogas tópicas ou sistêmicas, particularmente hipotensores sistêmicos usados por vários pacientes deste estudo. Porém, o fato destes medicamentos permanecerem inalterados antes e depois do uso da Brimonidina e do fato de não ter ocorrido alterações na PAS e na PAD, torna esta hipótese muito pouco provável.

A ação da Brimonidina pré-sináptica inibindo a liberação de catecolamina e assim inibindo o estímulo à vasoconstrição e promovendo talvez um "relaxamento passivo" poderia ser uma explicação para nossos resultados. Os receptores adrenérgicos alfa-2 pré-sinapticos, quando ativados, inibem a liberação de catecolamina por um mecanismo de feedback negativo, fisiologicamente. Quando estimulados pela Brimonidina, induziriam assim a redução na liberação de catecolamina pelo menos na íris ${ }^{(19-2)}$. Por isso a Brimonidina pode provocar miose ${ }^{(20-21)}$.

Como o Doppler colorido foi utilizado para medir as velocidades sangüíneas antes e depois do tratamento com Brimonidina, é importante que esta técnica forneça valores suficientemente reprodutíveis. Como foi demonstrado por Harris et al., $1995^{(22)}$, a reprodutibilidade das medidas da velocidade sangüínea na AO é alta $(12 \%, 6 \%$ e $4 \%$ para VSM, VDF e IR respectivamente). Esta grande reprodutibilidade demonstrada também por Costa et al., 1996 ${ }^{(23)}$, torna consistentes nossos resultados.

Neste estudo a Brimonidina reduziu a PIO em pacientes com glaucoma crônico em ambos os olhos de 9,9\% (17,20 para $15,50 \mathrm{mmHg})$ no OD e $9,3 \%$ (17,00 para $15,42 \mathrm{mmHg})$ no OE. Este decréscimo não foi porém significativo. 
A redução da $\mathrm{PIO}$ observada neste estudo foi inferior à observada por Lachkar et al., 1998 ${ }^{(10)},(17,7 \%)$ em pacientes portadores de hipertensão ocular, também inferior a queda de 4,7 mmHg observada por Toris et al., 1995(14), em voluntários com hipertensão ocular, à queda de 5,9 a 7,6 mmHg observada por Greenfield et al., $1997^{(3)}$, no pico, em pacientes com glaucoma e hipertensão ocular. Mas foi semelhante à redução de $1,8 \mathrm{mmHg}$ observada em homens jovens e saudáveis por Nordlund et al., $1995^{(24)}$.

A menor redução da PIO observada neste estudo pode ser causada por diferenças no tipo de pacientes estudados. Nossos pacientes eram portadores de glaucoma cujos tratamentos anteriores com 1 ou 2 drogas não eram satisfatórios. A maioria de nossos pacientes já usava betabloqueador tópico e segundo o estudo A 342-611 do Laboratório Allergan ${ }^{(25)}$, quando a Brimonidina é usada em associação com betabloqueador, a redução da PIO é menor do que a observada quando a Brimonidina é usada como droga única. Como o poder do teste foi de $16 \%$ para OD e $14 \%$ para OE, esta poderia ser outra explicação para a queda da PIO não ter sido significativa neste trabalho.

Nosso estudo confirma o efeito benéfico do tartarato de Brimonidina a $0,2 \%$ como tratamento único ou em associação, na redução da PIO e mostrou também efeito benéfico na VSM, VDF e IR na AO (indiretamente no fluxo sangüíneo) de pacientes com glaucoma crônico. Como não foi observado nenhum efeito nas PAS e PAD e foi observada redução da PIO, podemos dizer que provavelmente houve melhora da pressão de perfusão na cabeça do NO.

Outros estudos se fazem necessários para avaliação do efeito da Brimonidina (como tratamento único ou em associação) sobre a perfusão na cabeça do NO, particularmente em pacientes portadores de glaucoma crônico e em tratamentos crônicos.

Futuros aperfeiçoamentos nos métodos de avaliação do fluxo sangüíneo ocular (não somente velocidade de fluxo e índice de resistência), permitirão conclusões mais definitivas se a Brimonidina ou outras drogas utilizadas para reduzir a PIO, afetam também a circulação na cabeça do NO em pacientes com glaucoma.

\section{CONCLUSÃO}

O tartarato de Brimonidina a $0,2 \%$ aumentou significativamente a VSM e a VDF, e reduziu significativamente o IR na AO de pacientes portadores de glaucoma crônico. Este efeito sugere que a Brimonidina pode ser benéfica nos pacientes com glaucoma e insuficiência vascular na cabeça do NO.

\section{ABSTRACT}

Purpose: To evaluate a twice daily $0.2 \%$ brimonidine tartrate effect on retrobulbar circulation with color Doppler in glauco- matous patients. Methods: Both eyes of sixteen patients with glaucoma were studied. Using a Color Doppler imaging, peak systolic, end diastolic velocity and resistive index was determined in the ophthalmic artery, central retinal artery and temporal short posterior ciliary artery before and after $0.2 \%$ brimonidine treatment. Results: $0.2 \%$ brimonidine tartrate significantly improved peak systolic (28.24 to $34.23 \mathrm{~cm} / \mathrm{seg})$ and end diastolic velocities $(6.72$ to $8.10 \mathrm{~cm} / \mathrm{seg})$ in the right eye and decreased resistive index (0.75 to 0.71$)$ in the ophthalmic artery of the left eye. Conclusions: A twice daily $0.2 \%$ brimonidine tartrate treatment significantly increased peak systolic and end diastolic velocity and reduced resistive index in ophthalmic artery in patients with chronic glaucoma. This effect suggests that brimonidine could benefit glaucoma patients with optic nerve head vascular insufficiency.

Keywords: Optic disk/blood supply; Glaucoma; Quinoxalines/ therapeutic use; Color Doppler ultrasonography

\section{REFERÊNCIAS}

1. Hayreh SS. Progress in the understanding of the vascular etiology of glaucoma. Curr Opinion Ophthalmol 1994;5:26-35.

2. Fechtner RD, Weinreb RN. Mechanisms of optic nerve damage in primary open angle glaucoma. Surv Ophthalmol 1994;39:23-42.

3. Greenfield DS, Liebmann JM, Ritch R. Brimonidine: A new alpha ${ }_{2}$-adrenoreceptor agonist for glaucoma treatment. J Glaucoma 1997;6:250-7.

4. Barnebey H. Long-term efficacy of brimonidine on IOP lowering [abstract]. Invest Ophthalmol Vis Sci 1996;35:S1102.

5. Carter CJ, Brooks DE, Doyle DL, Drance SM. Investigations into a vascular etiology for low-tension glaucoma. [commented on Ophthalmology 1990;97: 838-9] Ophthalmology 1990;97:49-55.

6. Costa VP, Molnar LJ, Cerri GG. Avaliação da circulação retrobulbar através do Doppler colorido: estudo em indivíduos normais. Arq Bras Oftalmol 1996; 59:17-20.

7. Hayreh SS. The ophthalmic artery. III Branches. Br J Ophthalmol 1962;46: 212-47.

8. Hayreh SS, Beach KW. Optic nerve sheat decompression may improve blood flow optic neuropathy. Ophthalmology 1993;100:303-5; discussion.

9. Hansen NB, Stonestreet BS, Rosenkrantz TS, Oh W. Validity of Doppler measurements of anterior cerebral artery blood flow velocity: correlation with brain blood flow in piglets. Pediatrics 1983;72:526-31.

10. Lachkar Y, Migdal C, Dhanjil S. Effect of brimonidine tartrate on ocular hemodynamic measurements. Arch Ophthalmol 1998;116:1591-4.

11. Papaconstantinou DS, Georgopoulos GT, Andreanos DG, Vergados J, Patsea EE, Theodossiadis GP. Effect of topical Brimonidine on ocular blood flow [abstract]. Invest Ophthalmol Vis Science 2000;41:S551.

12. Carlsson AM, Chauhan BC, Lee AA, LeBlanc RP. The effect of brimonidine tartrate on retinal blood flow in patients with hypertension. Am J Ophthalmol 2000;129:297-301.

13. Spada CS, Nieves AL, Burke JA, Woodward DF. Comparative effects of brimonidine, p-aminoclonidine and clonidine on arteriolar caliber in human retinal tissue [abstract]. Invest Ophthalmol Vis Sci 1995;36:S1041.

14. Toris CB, Gleason ML, Camras CB, Yablonsky M. Effects of brimonidine on aqueous humor dynamics in human eyes. Arch Ophthalmol 1995;113:1514-7.

15. Bockman CS, Jeffries WB, Abel PW. Binding and functional characterizations of $\alpha-2$ adrenergic receptor subtypes on pig vascular endothelium. J Pharmacol Exp Ther 1993;267:1126-33.

16. Angus JA, Cocks TM, Wright CE, Satoh K, Campbell GR. Endothelium dependent responses in large arteries and in the microcirculation. In: Vanhoutte PM, editor Relaxing and contracting factors: biological and clinical research. Totowa NJ: Humana Press 1998. p. 361-87.

17. Yu DY, Alder VA, Su EN, Mele EM, Cringle SJ, Morgan WH. Agonist response of human isolated posterior ciliary artery. Invest Ophthalmol Vis Sci $1992 ; 33: 48-54$ 
18. Orgul S, Bacon DR, Van Buskirk EM, Cioffi GA. Optic nerve vasomotor effects of topical apraclonidine hydrochloride. Br J Ophthalmol 1996;80:82-4.

19. Hu PS, Fredholm BB. Alpha 2 - adrenoreceptor agonist - mediated inhibition of $[3 \mathrm{H}]$ noradrenaline release from rat hippocampus is reduced by 4 -aminopyridine, but that caused by an adenosine analogue or omega - conotoxin is not. Acta Physiol Scand 1989;136:347-53.

20. Burke J, Kharlamb A, Shan T, Runde E, Padillo E, Manlapaz C, et al. Adrenergic and imidazoline receptor - mediated responses to UK - 14, 30418 (brimonidine) in rabbits and monkeys. A species difference. Ann NY Acad Sci 1995;763:78-95.
21. Burke JA, Potter DE. Ocular effects of a relatively selective alpha 2 agonist (UK -14, 304-18) in cats, rabbits and monkeys. Curr Eye Res 1986;5:665-76.

22. Harris A, WIlliamson TH, Martin B, Shoemaker JA, Sergott RC, Spaeth GL, Katz JL. Test/retest reproducibility of color Doppler imaging assessment of blood flow velocity in orbital vessels. J Glaucoma 1995;4:281-6.

23. Costa VP, SuzukI R, Molnar LB, Cerri GG. A reprodutibilidade do exame de Doppler colorido. Rev Bras Oftalmol 1996;55:43-7.

24. Nordlund JR, Paquale LR, Robin AL, Rudikoff MT, Ordman J, Chen KS, Walt J. The cardiovascular, pulmonary and ocular hypotensive effects of $0.2 \%$ brimonidine. Arch Ophthalmol 1995;113:77-83.

25. Allergan-Study A 342-611. Alphagan Irvine, Calif: Allergan, s.d. p. 28-9.

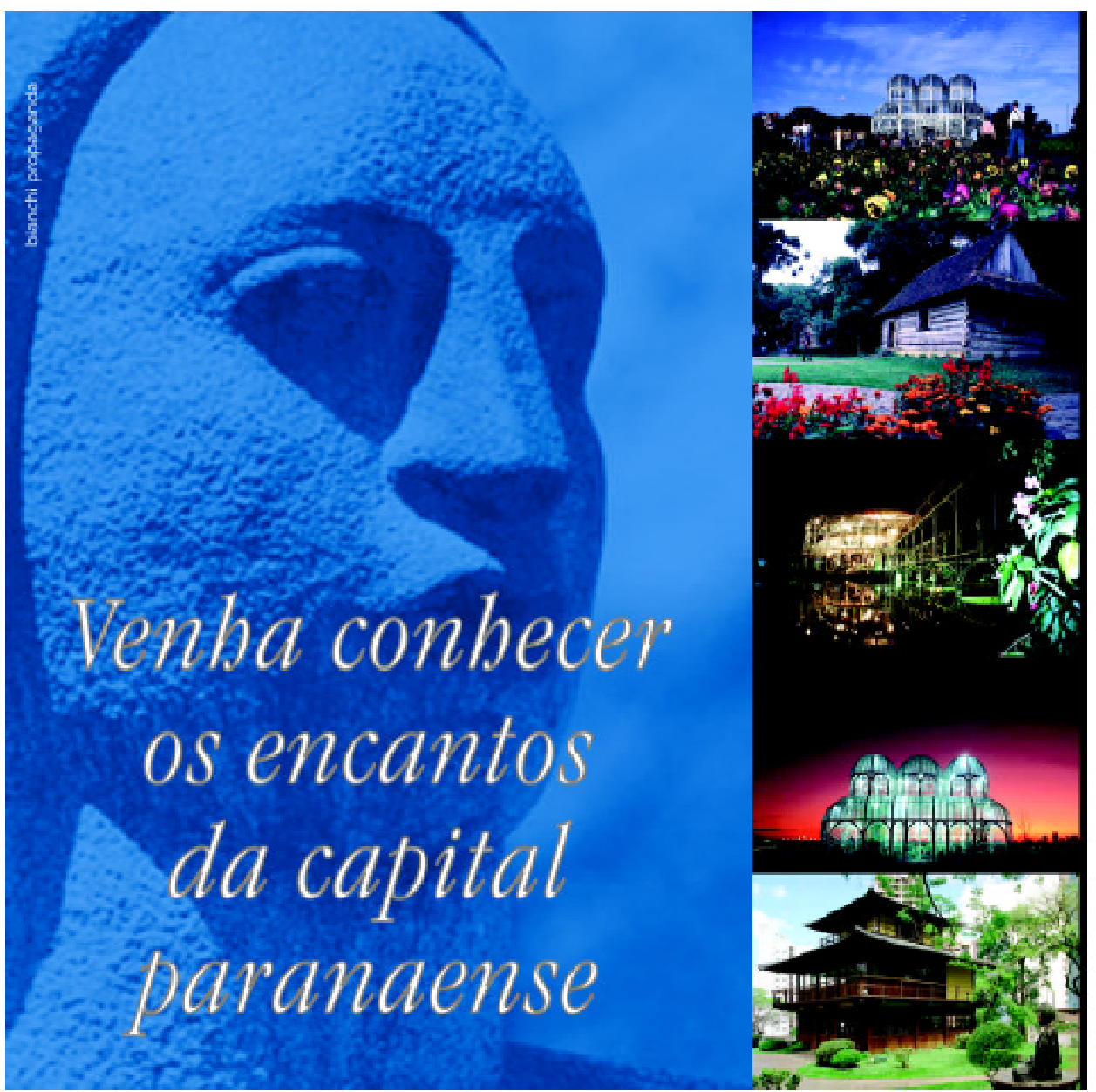

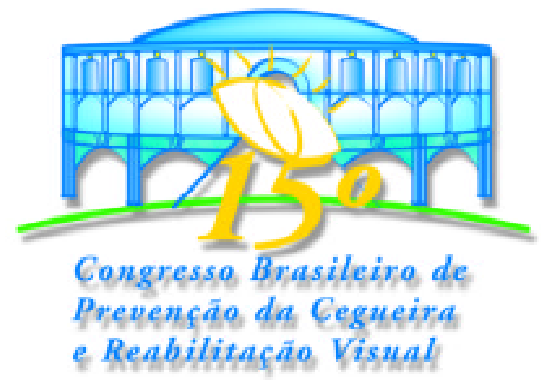

1 a 4 de setewho de 2002

Fino Trade Cinvitisa - PR

Informilcos: Th (11) 32664000 Fix: (11) 31710048

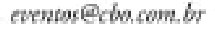

